\title{
Generation of cold Rydberg atoms at submicron distances from an optical nanofiber
}

\author{
Krishnapriya Subramonian Rajasree, ${ }^{1}$ Tridib Ray,,${ }^{1, *}$ Kristoffer Karlsson $\odot,{ }^{1}$ Jesse L. Everett, ${ }^{1}$ and Síle Nic Chormaic $\odot^{1,2, \dagger}$ \\ ${ }^{1}$ Light-Matter Interactions for Quantum Technologies Unit, \\ Okinawa Institute of Science and Technology Graduate University, Onna, Okinawa 904-0495, Japan \\ ${ }^{2}$ Université Grenoble Alpes, CNRS, Grenoble INP, Institut Néel, 38000 Grenoble, France
}

(Received 31 July 2019; accepted 20 January 2020; published 14 February 2020)

\begin{abstract}
We report on a controllable, hybrid quantum system consisting of cold Rydberg atoms and an optical nanofiber interface. Using a two-photon ladder-type excitation in ${ }^{87} \mathrm{Rb}$, we demonstrate both coherent and incoherent Rydberg excitation at submicron distances from the nanofiber surface. The 780-nm photon, near resonant to the $5 S \rightarrow 5 P$ transition, is mediated by the cooling laser, while the $482-\mathrm{nm}$ light, near resonant to the $5 P \rightarrow 29 D$ transition, is mediated by the guided mode of the nanofiber. The population loss rate of the cold atom ensemble is used to measure the Rydberg population rate. A theoretical model is developed to interpret the results and link the population loss rate to the experimentally measured, effective Rabi frequency of the process. This work makes headway in the study of Rydberg atom-surface interactions at submicron distances and the use of cold Rydberg atoms for all-fibered quantum networks.
\end{abstract}

DOI: 10.1103/PhysRevResearch.2.012038

In recent years, Rydberg atoms have emerged as leading candidates for neutral atom-based quantum information processing [1-4] and quantum simulations [5-7]. The long-lived quantum states and the precisely tunable dipolar interaction, leading to a blockade [8], can be used to prepare a mesoscopic atomic ensemble exhibiting quantum correlations and entanglement [9]. Such systems have already been used to demonstrate a quantum phase gate [10] and simulator [7] in free space. Interfacing interacting Rydberg atoms with microfabricated components is a very attractive choice for building compact and scalable hybrid quantum devices. Coherent Rydberg excitation has been reported for an atom chip [11], a $\mu \mathrm{m}$-sized vapor cell [12], a hollow-core photonic crystal fiber $[13,14]$, and has been proposed for a superconducting resonator [15], with each platform having its own advantages and disadvantages.

Here, we present an alternative, but highly viable, platform for atom-based quantum networks by interfacing cold Rydberg atoms with a single-mode optical nanofiber (ONF). To date, ground-state cold atom-ONF hybrid systems $[16,17]$ have shown tremendous potential for a new generation of quantum devices. The small cross section of the evanescent field from an ONF, as a result of the exponential radial decay from the fiber waist, leads to a large cooperativity $[18,19]$, the key to many quantum optics experiments. The high intensity

\footnotetext{
*Present address: Laboratoire Kastler Brossel, Sorbonne Université, CNRS, ENS-Université PSL, Collège de France, 4 place Jussieu, F-75005 Paris, France.

$\dagger$ sile.nicchormaic@ oist.jp

Published by the American Physical Society under the terms of the Creative Commons Attribution 4.0 International license. Further distribution of this work must maintain attribution to the author(s) and the published article's title, journal citation, and DOI.
}

and field gradient can be used to optically trap atoms in a one-dimensional array [20,21], thereby enabling the study of one-dimensional, many-body physics, or can be exploited for quadrupole transition enhancement [22]. Cooperative effects, such as the generation of a collective entangled state [23], have been demonstrated in such a system using ground-state neutral Cs atoms. In addition, atoms in the evanescent field region are intrinsically coupled to an optical bus in the form of the fiber-guided mode. This can lead to low-loss transfer of information to and from the interaction region [24], a prerequisite for Rydberg-based quantum repeaters in fibercoupled cavities [25].

Aside from endeavors to combine ONFs and ground-state neutral atoms, work on Rydberg excitation next to ONFs has, to date, been limited to theoretical proposals due to the difficulty in generating highly excited atom states within a few $100 \mathrm{~nm}$ of surfaces, e.g., dielectrics or metals, and the problem of induced electric fields - even at distances as large as $\sim 100 \mu \mathrm{m}$ - by adsorption of atoms on the surface $[26,27]$. In this Rapid Communication, we report on evanescent fieldassisted Rydberg excitation at submicron distances from the surface of an ONF, which is embedded in a ${ }^{87} \mathrm{Rb}$ atomic ensemble in a magneto-optical trap (MOT). A ladder-type, two-photon excitation scheme [28] is used to excite the atoms to the Rydberg state and a trap loss method [29] is used to probe the Rydberg excitation. We implement a rate equation model [30] to determine the rate of population transfer to the Rydberg state. Both coherent two-photon excitation and incoherent two-step excitation is demonstrated. A density matrix-based model is developed for the three-level laddertype system interacting with the evanescent field of the ONF. The developed model explains the main features of our experimental results.

The experiment consists of an ONF, with a waist diameter of $\sim 400 \mathrm{~nm}$, that is single mode for $780 \mathrm{~nm}$, embedded in a cold atomic ensemble of ${ }^{87} \mathrm{Rb}$. A schematic of the 


$$
\text { (a) }
$$
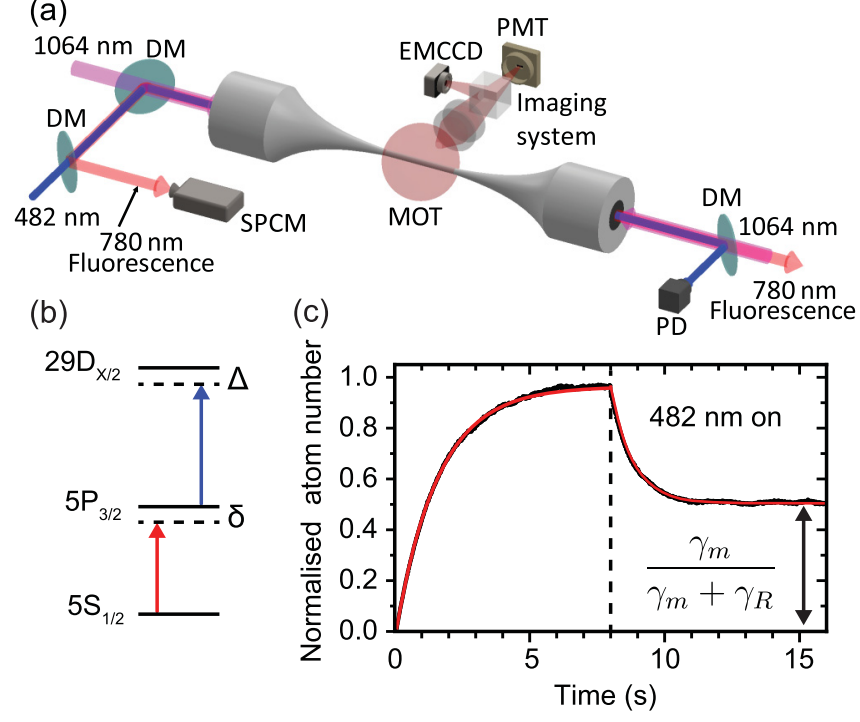

FIG. 1. (a) Experimental setup. DM: Dichroic mirror. PD: Photodiode. PMT: Photomultiplier tube. EMCCD: Electron multiplying charge-coupled device. SPCM: Single-photon counting module. Imaging system: Lens and beam-splitter combination. (b) Simplified ${ }^{87} \mathrm{Rb}$ energy diagram. Atoms trapped in the MOT are excited to the $n=29$ level by a two-photon process involving the cooling laser at 780- and 482-nm light in the evanescent field of the ONF. (c) Typical loading of the MOT to a steady state for $8 \mathrm{~s}$ and subsequent decay to a new equilibrium for $8 \mathrm{~s}$ after the 482-nm laser has been switched on.

experimental setup is given in Fig. 1(a). The ONF is fabricated by exponentially tapering a section of SM800125 fiber (cutoff wavelength $697 \mathrm{~nm}$ ) using a $\mathrm{H}: \mathrm{O}$ flamebrushing technique [31]. To guarantee a low-loss ONF in the ultrahigh vacuum (UHV) chamber, we ensure that the tapering process is adiabatic and that the fiber itself is very clean. During the experiment, $100 \mu \mathrm{W}$ of 1064-nm light is passed through the ONF from each side to keep the fiber hot and avoid atom deposition on the ONF [32]. This light has the added advantage of attracting atoms toward the ONF surface, thereby increasing the number of atoms interacting with the evanescent field.

The ${ }^{87} \mathrm{Rb}$ atoms are cooled to $\sim 120 \mu \mathrm{K}$ using a standard MOT configuration of three pairs of retroreflected cooling and repump beams. The $780-\mathrm{nm}$ cooling beam is $14 \mathrm{MHz}$ reddetuned from the $5 S_{1 / 2}(F=2) \rightarrow 5 P_{3 / 2}\left(F^{\prime}=3\right)$ transition and the repump is on resonance with the $5 S_{1 / 2}(F=1) \rightarrow$ $5 P_{3 / 2}\left(F^{\prime}=2\right)$ transition. The total powers in the cooling and repump beams are 42 and $2 \mathrm{~mW}$, respectively. Using a magnetic field gradient of $\sim 24 \mathrm{G} / \mathrm{cm}$ at the center of the MOT, typically $10^{7}$ atoms are trapped, and the typical Gaussian full width at half maximum (FWHM) of the MOT is $0.5 \mathrm{~mm}$. The free-space MOT fluorescence is collected by an achromatic doublet ( $f=150 \mathrm{~mm}$ ) and is divided into two parts using a 50:50 beam splitter. One half of the signal is collected by a PMT to measure the instantaneous number of atoms in the MOT. The other half is imaged by an EMCCD camera to obtain the atom cloud density profile. The MOT fluorescence that couples to the guided mode of the ONF is separated from light of other wavelengths using an assembly of dichroic mirrors and bandpass filters, before being delivered to an SPCM. The overlap between the cold atom cloud and the ONF is optimized by maximizing the photon count at the SPCM using three pairs of Helmholtz coils. With an optimized overlap, the SPCM count is proportional to the atom density, and hence the PMT signal, for a given Gaussian FWHM.

The Rydberg excitation is driven by a ladder-type, twophoton process, shown in Fig. 1(b). The 780-nm light is provided by the cooling beams, while the 482-nm light is derived from a Toptica TA SHG Pro system and coupled into the nanofiber. The frequency of the 482-nm laser is stabilized to a vapor cell electromagnetically induced transparency (EIT) signal [28,33]. However, we implement a modification using an electro-optic modulator (EOM) to shift the frequency. First, the $780-n m$ probe laser is locked to the $5 S_{1 / 2}(F=2) \rightarrow$ $5 P_{3 / 2}\left(F^{\prime}=2,3\right)_{\text {co }}$ transition of ${ }^{85} \mathrm{Rb}$. The EOM is used to generate sidebands at $1.06632 \mathrm{GHz}$, one of which is resonant with the ${ }^{87} \mathrm{Rb} 5 S_{1 / 2}(F=2) \rightarrow 5 P_{3 / 2}\left(F^{\prime}=3\right)$ transition. In the ${ }^{87} \mathrm{Rb}$-enriched vapor cell, only the resonant sideband participates in the EIT process and the resultant EIT peak is used to lock the 482-nm laser frequency, which can now be adjusted simply by changing the frequency of the sideband.

The 482-nm light can be switched on and off using a combination of an acousto-optic modulator (AOM) and a mechanical shutter to ensure complete cutoff. This light is coupled to the ONF using a pair of dichroic mirrors (DMLP650) and interacts with the atoms in the MOT via the evanescent field. The coupling efficiencies for light of different wavelengths into the fiber patch cable differ, as do the losses at any splice regions, making it difficult to determine the exact transmission through the ONF for each wavelength used in the experiment. We therefore measure the power at the output pigtail of the ONF in order to estimate the power at the nanofiber waist. Unless another power is explicitly mentioned, we maintain an output power of $16 \mu \mathrm{W}$ for the 482-nm light for all measurements. Note that, at $482 \mathrm{~nm}$, the ONF supports the fundamental mode $\mathrm{HE}_{11}$ as well as the $\mathrm{TE}_{01}, \mathrm{TM}_{01}$, and $\mathrm{HE}_{21}$ higher-order modes. The ONF is single mode for all other wavelengths used.

The experimental sequence is simple. First, the MOT is loaded to saturation for $8 \mathrm{~s}$. The population of the MOT at any time $t$, as a fraction of the undisturbed equilibrium population, can be obtained from the PMT signal and is expressed as

$$
N(t)=\frac{N_{\text {tot }}(t)}{N_{0}}=\left(1-e^{-\gamma_{m} t}\right),
$$

where $N_{\text {tot }}(t)$ is the total number of atoms in the MOT at time $t$ and $N_{0}=L / \gamma_{m}$ is the steady-state number of atoms in the MOT. $L$ is the loading rate of atoms into the MOT from the background vapor and $\gamma_{m}$ is the population loss rate of the MOT. A typical loading curve is shown in Fig. 1(c) (left-hand side of the plot) and $\gamma_{m}$ is obtained by fitting the PMT signal to Eq. (1).

When the MOT is loaded to saturation, the 482-nm laser propagating in the ONF is turned on. Only those atoms in the evanescent field of the nanofiber can interact with both the 780- and 480-nm light and therefore participate in the two-photon Rydberg excitation. The newly formed Rydberg atoms leave the cooling cycle and escape from the MOTthis introduces an additional population loss rate $\gamma_{R}$, which 


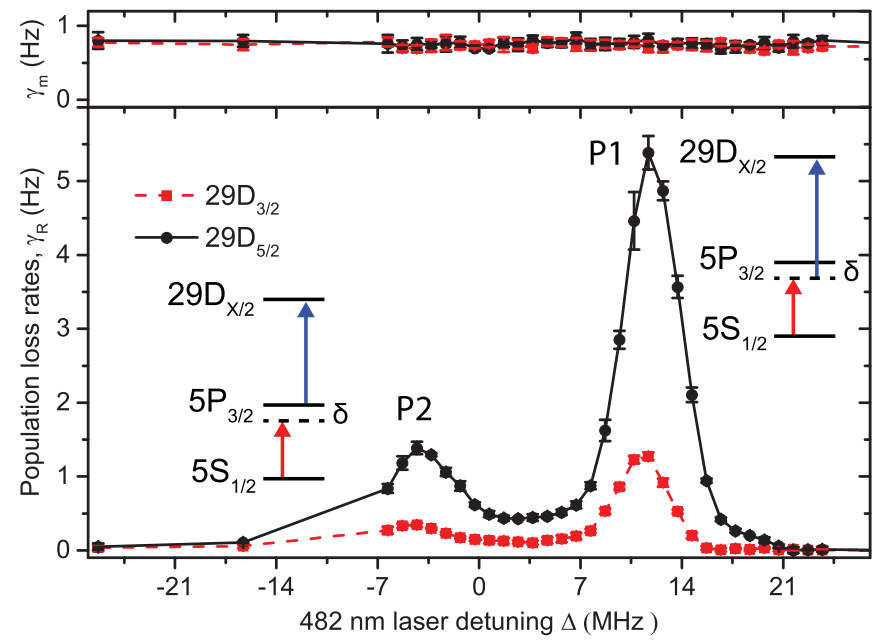

FIG. 2. Dependence of the population loss rates, $\gamma_{m}$ (top) and $\gamma_{R}$ (bottom), on the 482-nm laser detuning $\Delta$ for a fixed detuning of $\delta=14 \mathrm{MHz}$ for the 780-nm laser. For both levels, 29D $D_{3 / 2}$ and $29 D_{5 / 2}, \gamma_{m}$ does not change while $\gamma_{R}$ shows two peaks. The peaks are labeled with the level schemes to indicate the coherent (P1) and the incoherent $(\mathrm{P} 2)$ processes [34].

includes any other loss processes, such as ionization of atoms post Rydberg excitation. This loss mechanism starts bleeding the MOT of atoms and a second equilibrium is established over time, determined by the total loss rate $\gamma_{t}=\gamma_{m}+\gamma_{R}$. The time-dependent population of the MOT can be written as

$$
N(t)=1-\frac{\gamma_{R}}{\gamma_{t}}\left(1-e^{-\gamma_{t}\left(t-t_{0}\right)}\right),
$$

where $t_{0}$ is the time at which the 482-nm laser is switched on. The time evolution of the MOT population is fitted to Eq. (2) to obtain $\gamma_{R}$. Assuming all atoms excited to the Rydberg state are lost from the cooling cycle, $N_{0} \gamma_{R}$ is the rate of formation of the Rydberg atoms at the moment the 482-nm laser is switched on.

For a given detuning $\Delta$ of the 482 -nm laser from the $5 P_{3 / 2}$ to the $29 D$ Rydberg level, the experiment is repeated for eight cycles to obtain mean values of $\gamma_{m}$ and $\gamma_{R}$. The variation of $\gamma_{m}$ and $\gamma_{R}$ as a function of $\Delta$ is investigated for two Rydberg levels, namely, $29 D_{5 / 2}$ and $29 D_{3 / 2}$. The results are presented in Fig. 2. Note that $\gamma_{m}$ does not change during the experiment, ensuring that the experimental conditions also do not change. We can clearly see that $\gamma_{R}$ shows two peaks as a function of $\Delta$ for both of the $29 D$ levels. The two peaks, i.e., P1 at $\Delta=$ $11.7 \mathrm{MHz}$ and $\mathrm{P} 2$ at $\Delta=-4.3 \mathrm{MHz}$, correspond to two different mechanisms for the Rydberg excitation. P1 is obtained from a coherent, two-photon excitation, where a fraction of the ground-state atom population is coherently transferred to the Rydberg state without populating the intermediate, $P_{3 / 2}$, excited state. In contrast, P2 corresponds to an incoherent, two-step excitation process. The cooling laser transfers a fraction of the ground-state population to the intermediate state, $P_{3 / 2}$. The second photon, at $482 \mathrm{~nm}$, then transfers a fraction of the $P_{3 / 2}$ population to the Rydberg state. In this process, the intermediate state is populated and there is no coherence established between the ground and Rydberg states. A detailed explanation of the mechanism affecting the ratio

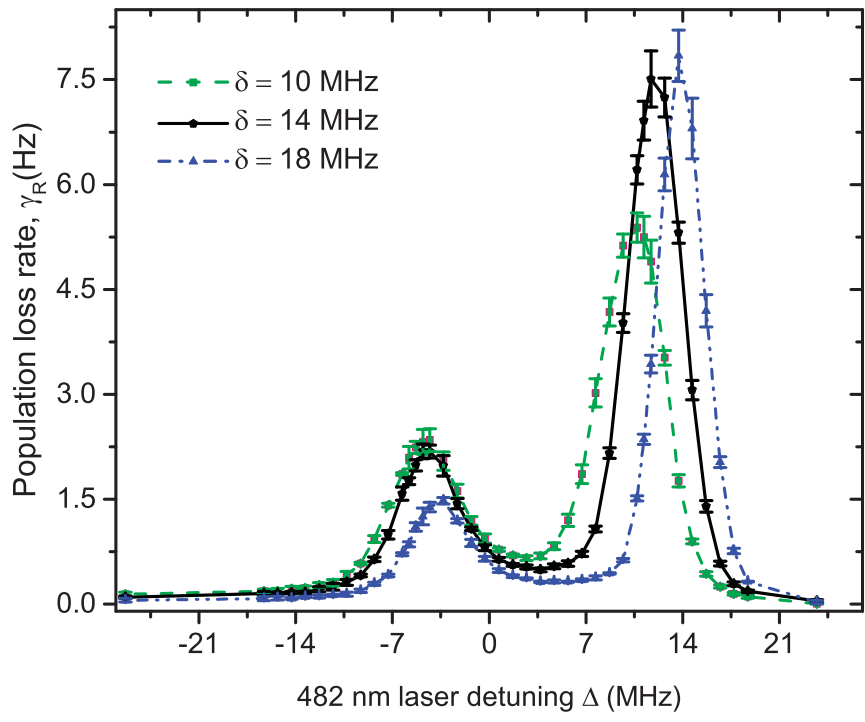

FIG. 3. Dependence of the population loss rate $\gamma_{R}$ on the 482-nm laser detuning $\Delta$ for three different detunings of $780 \mathrm{~nm}$, i.e., $\delta=10$, 14 , and $18 \mathrm{MHz}$, for the $29 D_{5 / 2}$ level.

between coherent and incoherent excitations can be found in Ref. [34]. Ideally, the peaks should appear at $\Delta=0$ and $\delta$ (the detuning of the 780-nm cooling laser). The deviation of the peaks from the expected position and separation values may arise from many factors, such as a light shift, a van der Waal's shift due to the fiber surface [20,35-37], or a shift due to stray electric charge on the fiber surface [14], experienced by the energy levels involved in the excitation process. These effects have not been incorporated in the model presented herein.

Figure 3 shows the variation of $\gamma_{R}$ as a function of $\Delta$, for three different values of the cooling laser detuning $\delta$. The position of the coherent peak P1 changes with $\delta$ to satisfy the two-photon resonance condition. In this process, the sum of the energies of the 780-nm photon and the 482-nm photon should be equal to the energy difference between the ground and Rydberg state. The $5 P_{3 / 2}$ intermediate state is always populated following a small change in detuning of the cooling laser. Therefore, the peak position should not change since the 482-nm photon should always have the same energy in order to couple the $5 P_{3 / 2}$ intermediate state to the Rydberg level; however, this argument ignores other effects that could lead to energy level shifts in the experiment. As we expect, the position of $\mathrm{P} 2$ does not noticeably change for $\delta=10$ and $14 \mathrm{MHz}$; however, there is a small, observable shift when $\delta=$ $18 \mathrm{MHz}$. A model accounting for surface and charge effects in a more realistic geometry would be needed to determine the reason for this observed shift.

Finally, we investigated the dependence of $\gamma_{R}$ on the effective Rabi frequency of the $5 P_{3 / 2} \rightarrow 29 D_{X / 2}$ transition for both the coherent and incoherent processes. To perform this experiment, $\delta$ was set to $14 \mathrm{MHz}$ and $\Delta$ was set to the maximum of either $\mathrm{P} 1$ or $\mathrm{P} 2$. The power of the 482 -nm laser was then varied and $\gamma_{R}$ was measured for both peaks and both transitions. The results are shown in Fig. 4. We compare the dependence of the observed loss rates on the frequencies and intensities of the driving optical fields by considering a three-level density 


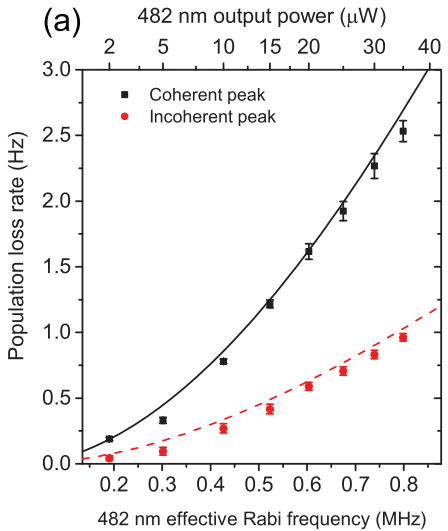

(b) $482 \mathrm{~nm}$ output power $(\mu \mathrm{W})$

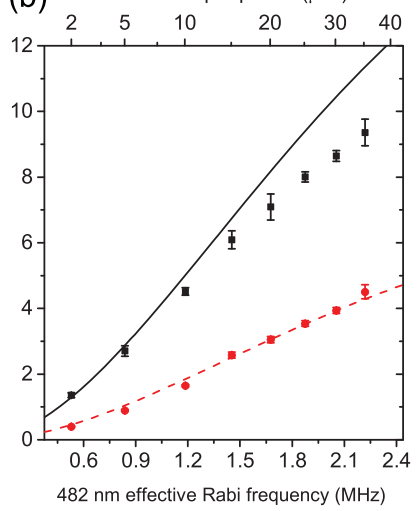

FIG. 4. The dependence of $\gamma_{R}$ for the coherent (P1 black) and incoherent (P2 red) excitation on the power of the 482-nm pump laser and hence the effective Rabi frequency for (a) $29 D_{3 / 2}$ and (b) $29 D_{5 / 2}$. The solid lines are solutions to the three-level model.

matrix model for a population of atoms driven by two coherent optical fields of constant intensity. We use the Maxwell-Bloch equations for thermal atoms in a small interaction volume. As the atoms interact with different intensities of the evanescent field at different distances from the ONF surface, the Rabi frequency is position dependent. We define an effective Rabi frequency $\Omega_{r}$ averaged over the atom ensemble interacting with the evanescent field for the $5 P_{3 / 2} \rightarrow 29 D_{X / 2}$ transition. Using the definitions of the Rabi frequency $\Omega_{p}$ for the cooling transition, the effective Rabi frequency $\Omega_{r}$ for the Rydberg transition, detunings from resonance $\Delta_{p}$ and $\Delta_{r}$, and atomic operators $\sigma_{i j}$, representing populations and coherences of all three levels, we can write

$$
\begin{aligned}
\partial_{t} \sigma_{s s}= & {\left[i \frac{\Omega_{p}}{2} \sigma_{p s}+\text { H.c. }\right]+\Gamma_{p} \sigma_{p p}+\Gamma_{r} \sigma_{r r}-A\left(\sigma_{s s}-\frac{1}{2}\right), } \\
\partial_{t} \sigma_{p p}= & {\left[i\left(\frac{\Omega_{r}}{2} \sigma_{r p}-\frac{\Omega_{p}}{2} \sigma_{p s}\right)+\text { H.c. }\right]-\Gamma_{p} \sigma_{p p} } \\
& -A\left(\sigma_{p p}-\frac{1}{2}\right), \\
\partial_{t} \sigma_{r r}= & {\left[i \frac{\Omega_{r}}{2} \sigma_{r p}+\text { H.c. }\right]-\left(\Gamma_{r}+A\right) \sigma_{r r}, } \\
\partial_{t} \sigma_{p s}= & -i \Delta_{p} \sigma_{p s}+i \frac{\Omega_{p}}{2}\left(\sigma_{s} s-\sigma_{p} p\right)+i \frac{\Omega_{r}}{2} \sigma_{r s} \\
& -\left(\frac{\Gamma_{p}}{2}+A\right) \sigma_{p s}, \\
\partial_{t} \sigma_{r p}= & -i \Delta_{r} \sigma_{r p}+i \frac{\Omega_{r}}{2}\left(\sigma_{p p}-\sigma_{r r}\right)-i \frac{\Omega_{r}}{2} \sigma_{r s} \\
& -\left(\frac{\Gamma_{p}+\Gamma_{r}}{2}+A\right) \sigma_{r p}, \\
& -\left(\frac{\Gamma_{r}}{2}+A+\gamma_{0}\right) \sigma_{r s} . \\
\partial_{t} \sigma_{r s}= & -i\left(\Delta_{p}+\Delta_{r}\right) \sigma_{r p}+i \frac{\Omega_{r}}{2} \sigma_{p s}-i \frac{\Omega_{p}}{2} \sigma_{r p} \\
& -(i)
\end{aligned}
$$

Here, $\gamma_{0}$ is the motional dephasing of the coherence $\sigma_{r s}$. For a MOT temperature of $120 \mu \mathrm{K}$ and a coherence generated by a cooling beam perpendicular to the nanofiber axis, $\gamma_{0}=$ $600 \pm 200 \mathrm{kHz}$. Other motional dephasings are ignored. The + H.c. terms indicate that the Hermitian conjugates of the terms in the brackets [ ] are also included. The thermal motion of atoms into and out of the interaction volume removes atoms from the populations and coherences at a rate $A$ and places them in a mixture of roughly $s \sigma_{s s}+p \sigma_{p p}$, with $s$ and $p$ determined by $\Omega_{p}$ and $\Delta_{p}$. The model ignores other aspects of the experimental geometry. $\Omega_{p}$ is fitted from the splitting of $\mathrm{P} 1$ and $\mathrm{P} 2$, and $\Omega_{r}$ is fitted to the $\mathrm{P} 1$ and $\mathrm{P} 2$ height data. The decoherence rates are fitted to the widths of $\mathrm{P} 1$ and $\mathrm{P} 2$.

The mixing rate $A$ is an important parameter for describing this experiment. The introduction of atoms in a mixed state to the interaction region boosts the incoherent production of Rydberg atoms well beyond that expected in a coherently driven system. The mixing rate may be changed experimentally by changing the cooling field detuning and thus the temperature of the atoms. The effect of this can be seen in Fig. 3, where a closer detuned trap with hotter atoms has a higher mixing rate and a larger incoherent peak to coherent peak height ratio than a further detuned, cooler trap.

The population of Rydberg atoms $\sigma_{r r}$ in the model is related to the experimental loss rate $\gamma_{R}$ by estimating the proportion $P$ of atoms in the MOT that are also in the evanescent field and multiplying by the mixing rate: $P A \sigma_{r r} \approx \gamma_{R}$. The value of $P$ is set to $P=(3 \pm 1) \times 10^{-4}$ to fit the model to the experimental data in Fig. 4 , and this value also agrees with other experimental observations. It corresponds to an interaction region extending about $100-200 \mathrm{~nm}$ from the fiber surface, noting that the $1 / e$ decay length of the evanescent field of the fundamental mode of $482-\mathrm{nm}$ light is $125 \mathrm{~nm}$. The mixing rate $A$ is set to $0.6 \mathrm{MHz}$ to fit the ratio of the incoherent to coherent peak heights in Fig. 4. This value is also consistent with the average flight time of atoms at $120 \mu \mathrm{K}$ through an interaction region with a diameter $d=$ $200 \mathrm{~nm}$, where $A \approx \bar{v} / d$, with $\bar{v}$ the average speed of the atoms.

The experimental loss rate clearly follows the theoretical curve at low $\gamma_{R}$, but diverges from the model above rates of $5 \mathrm{~Hz}$. This may be due to production of Rydberg atoms beginning to saturate as $\sigma_{r r} \rightarrow 1$. This is not observed in the model, with a Rydberg population at $\mathrm{P} 1$ of $\sigma_{r r}=0.033$ for $\Omega_{r}=1.25 \mathrm{MHz}$, which is well below saturation. The model only includes one cooling field, with a Rabi frequency equal to that of one cooling beam. $\Omega_{r}$ is assumed to be constant, whereas, in the experiment, atoms are subject to a timevarying interaction as they travel through the evanescent field. Averaging over these trajectories may give a more accurate relationship between the $482-\mathrm{nm}$ intensity and the effective $\Omega_{r}$. The model also assumes that all atoms leaving the interaction region while in the Rydberg state are lost from the MOT. However, considering the lifetime of the Rydberg states and the temperature of the atoms, a significant proportion of these atoms could actually be recaptured directly into the MOT if they decay into the cooling cycle. Experimental determination of the saturated loss rates under different conditions and for different $n$ levels could allow any processes interfering with recapture, such as ionization, to be observed. However, despite its simplicity, the model explains most of our observations and confirms the production of Rydberg atoms in the evanescent field of the nanofiber. 
In summary, we have reported on the generation of $n=29$ level Rydberg atoms in a ${ }^{87} \mathrm{Rb}$ cold atom ensemble surrounding an optical nanofiber and have shown that this is a very viable system for hybrid atom-nanofiber devices. By comparing the loss rate to the Rydberg population in a simple three-level density matrix model, we were able to closely model the experimental data and determine the size of the interaction region. Excitation of the Rydberg atoms is mediated via the optical nanofiber and they are estimated to be generated at no more than a couple of $100 \mathrm{~nm}$ from the surface where overlap between the two excitation fields (780 and $482 \mathrm{~nm}$ ) is maximum. This is an important advance on earlier experiments related to Rydberg atom generation close to dielectric surfaces $[26,27]$ and opens up many avenues of research such as all-fibered quantum networks using Rydberg atoms, excited atom-surface interactions at submicron distances, including van der Waal's interactions, effects on the Rydberg blockade or facilitation phenomena [38] in this regime, stray electric field effects from the dielectric nanofiber on energy levels and lifetimes of the atom, and the limitation of the maximum excited state ( $n$ value) that can be generated close to the nanofiber due to the atom size increasing quadratically with $n$. An estimate of the electric field present and its influence on the Rydberg atom lifetime is highly desirable and will be the focus of the next generation of experiments. For now, we can assume that the charge per unit length of the tapered fiber is no more than $2 \times 10^{11} \mathrm{C} / \mathrm{m}$ [39]. This number could increase when light propagates through the fiber [40], but the polarizability of the $29 D$ state indicates that the field must be limited to only a few $\mathrm{V} / \mathrm{cm}$ in order to generate the Rydberg atoms at the right laser detuning. A systematic study related to the Rydberg atom formation and lifetime as a function of power in the blue beam is also desirable. The observed redshifts on the resonance frequencies are assumed to be dominated by van der Waals interactions between the atoms and the fiber surface [35-37].

The versatility of this atom-nanofiber hybrid system could be extended to explore three-step Rydberg excitations [41] where the fiber would be single mode for the light used to drive the atomic transitions. Hence, the mode overlap in the evanescent field would be increased and Rydberg generation efficiency should be improved. A loss in detection of the $420-\mathrm{nm}$ light in the $6 P \rightarrow 5 S$ decay channel could also provide an alternative, nondestructive mechanism for Rydberg atom detection. In addition, a comprehensive study of the coherent interactions in MOT-embedded nanofibers could extend this experimental technique beyond a qualitative confirmation towards an investigation of the behavior of Rydberg or other exotic states, e.g., Rydberg polarons [42], adjacent to optical nanofibers. Future work will focus on trapping atoms at defined distances from the nanofiber to explore limitations on $n$ and on a study of the influence of the nanofiber on Rydberg levels using EIT signals [28].

This work was supported by Okinawa Institute of Science and Technology Graduate University, Japan Society for the Promotion of Science Postdoctoral Fellowship for Overseas Researchers Grant No. JPPE16769, and Grant-in-Aid for Scientific Research (C) Grant No. 19K05316.

The authors acknowledge early contributions by M. Langbecker and useful discussions with E. Stourm, J. Robert, E. Brion, and R. Löw. K.S.R., T.R., and K.K. conducted the experiments. J.E. did the theoretical modeling. S.N.C. and T.R. conceptualized the work. All authors wrote the manuscript.
[1] D. Jaksch, J. I. Cirac, P. Zoller, S. L. Rolston, R. Côté, and M. D. Lukin, Fast Quantum Gates for Neutral Atoms, Phys. Rev. Lett. 85, 2208 (2000).

[2] M. D. Lukin, M. Fleischhauer, R. Côté, L. M. Duan, D. Jaksch, J. I. Cirac, and P. Zoller, Dipole Blockade and Quantum Information Processing in Mesoscopic Atomic Ensembles, Phys. Rev. Lett. 87, 037901 (2001).

[3] M. Saffman, T. G. Walker, and K. Mølmer, Quantum information with Rydberg atoms, Rev. Mod. Phys. 82, 2313 (2010).

[4] C. S. Adams, J. D. Pritchard, and J. P. Shaffer, Rydberg atom quantum technologies, J. Phys. B: At., Mol. Opt. Phys. 53, 012002 (2019).

[5] H. Weimer, M. Müller, I. Lesanovsky, P. Zoller, and H. P. Büchler, A Rydberg quantum simulator, Nat. Phys. 6, 382 (2010).

[6] H. Labuhn, D. Barredo, S. Ravets, S. de Léséleuc, T. Macrì, T. Lahaye, and A. Browaeys, Tunable two-dimensional arrays of single Rydberg atoms for realizing quantum Ising models, Nature (London) 534, 667 (2016).

[7] H. Bernien, S. Schwartz, A. Keesling, H. Levine, A. Omran, H. Pichler, S. Choi, A. S. Zibrov, M. Endres, M. Greiner, V. Vuletić, and M. D. Lukin, Probing many-body dynamics on a 51-atom quantum simulator, Nature (London) 551, 579 (2017).
[8] D. Tong, S. M. Farooqi, J. Stanojevic, S. Krishnan, Y. P. Zhang, R. Côté, E. E. Eyler, and P. L. Gould, Local Blockade of Rydberg Excitation in an Ultracold Gas, Phys. Rev. Lett. 93, 063001 (2004).

[9] T. Wilk, A. Gaëtan, C. Evellin, J. Wolters, Y. Miroshnychenko, P. Grangier, and A. Browaeys, Entanglement of Two Individual Neutral Atoms Using Rydberg Blockade, Phys. Rev. Lett. 104, 010502 (2010).

[10] L. Isenhower, E. Urban, X. L. Zhang, A. T. Gill, T. Henage, T. A. Johnson, T. G. Walker, and M. Saffman, Demonstration of a Neutral Atom Controlled-NOT Quantum Gate, Phys. Rev. Lett. 104, 010503 (2010).

[11] J. de Hond, R. van Bijnen, S. J. J. M. F. Kokkelmans, R. J. C. Spreeuw, H. B. van Linden van den Heuvell, and N. J. van Druten, From coherent collective excitation to Rydberg blockade on an atom chip, Phys. Rev. A 98, 062714 (2018).

[12] H. Kübler, J. P. Shaffer, T. Baluktsian, R. Löw, and T. Pfau, Coherent excitation of Rydberg atoms in micrometre-sized atomic vapour cells, Nat. Photonics 4, 112 (2010).

[13] G. Epple, K. Kleinbach, T. Euser, N. Joly, T. Pfau, P. S. J. Russell, and R. Löw, Rydberg atoms in hollow-core photonic crystal fibres, Nat. Commun. 5, 4132 (2014). 
[14] M. Langbecker, M. Noaman, N. Kjærgaard, F. Benabid, and P. Windpassinger, Rydberg excitation of cold atoms inside a hollow-core fiber, Phys. Rev. A 96, 041402(R) (2017).

[15] D. Yu, L. C. Kwek, L. Amico, and R. Dumke, Superconducting qubit-resonator-atom hybrid system, Quantum Sci. Technol. 2, 035005 (2017).

[16] T. Nieddu, V. Gokhroo, and S. Nic Chormaic, Optical nanofibres and neutral atoms, J. Opt. 18, 053001 (2016).

[17] P. Solano, J. A. Grover, J. E. Hoffman, S. Ravets, F. K. Fatemi, L. A. Orozco, and S. L. Rolston, Optical nanofibers: A new platform for quantum optics, in Advances in Atomic, Molecular, and Optical Physics, edited by E. Arimondo, C. C. Lin, and S. F. Yelin, Vol. 66 (Academic, New York, 2017), Chap. 7, pp. 439-505.

[18] F. Le Kien, S. Dutta Gupta, V. I. Balykin, and K. Hakuta, Spontaneous emission of a cesium atom near a nanofiber: Efficient coupling of light to guided modes, Phys. Rev. A 72, 032509 (2005).

[19] F. Le Kien, V. I. Balykin, and K. Hakuta, Scattering of an evanescent light field by a single cesium atom near a nanofiber, Phys. Rev. A 73, 013819 (2006).

[20] F. Le Kien, V. I. Balykin, and K. Hakuta, Atom trap and waveguide using a two-color evanescent light field around a subwavelength-diameter optical fiber, Phys. Rev. A 70, 063403 (2004).

[21] E. Vetsch, D. Reitz, G. Sagué, R. Schmidt, S. T. Dawkins, and A. Rauschenbeutel, Optical Interface Created by LaserCooled Atoms Trapped in the Evanescent Field Surrounding an Optical Nanofiber, Phys. Rev. Lett. 104, 203603 (2010).

[22] F. Le Kien, T. Ray, T. Nieddu, T. Busch, and S. Nic Chormaic, Enhancement of the quadrupole interaction of an atom with the guided light of an ultrathin optical fiber, Phys. Rev. A 97, 013821 (2018).

[23] N. V. Corzo, J. Raskop, A. Chandra, A. S. Sheremet, B. Gouraud, and J. Laurat, Waveguide-coupled single collective excitation of atomic arrays, Nature (London) 566, 359 (2019).

[24] E. Stourm, Y. Zhang, M. Lepers, R. Guérout, J. Robert, S. Nic Chormaic, K. Mølmer, and E. Brion, Spontaneous emission of a sodium Rydberg atom close to an optical nanofibre, J. Phys. B: At., Mol. Opt. Phys. 52, 045503 (2019).

[25] E. Brion, F. Carlier, V. M. Akulin, and K. Mølmer, Quantum repeater with Rydberg-blocked atomic ensembles in fiber-coupled cavities, Phys. Rev. A 85, 042324 (2012).

[26] J. A. Sedlacek, E. Kim, S. T. Rittenhouse, P. F. Weck, H. R. Sadeghpour, and J. P. Shaffer, Electric Field Cancellation on Quartz by Rb Adsorbate-Induced Negative Electron Affinity, Phys. Rev. Lett. 116, 133201 (2016).

[27] A. Tauschinsky, R. M. T. Thijssen, S. Whitlock, H. B. van Linden van den Heuvell, and R. J. C. Spreeuw, Spatially resolved excitation of Rydberg atoms and surface effects on an atom chip, Phys. Rev. A 81, 063411 (2010).
[28] A. K. Mohapatra, T. R. Jackson, and C. S. Adams, Coherent Optical Detection of Highly Excited Rydberg States Using Electromagnetically Induced Transparency, Phys. Rev. Lett. 98, 113003 (2007).

[29] J. O. Day, E. Brekke, and T. G. Walker, Dynamics of lowdensity ultracold Rydberg gases, Phys. Rev. A 77, 052712 (2008).

[30] S. Lee, K. Ravi, and S. A. Rangwala, Measurement of collisions between rubidium atoms and optically dark rubidium ions in trapped mixtures, Phys. Rev. A 87, 052701 (2013).

[31] J. M. Ward, A. Maimaiti, V. H. Le, and S. Nic Chormaic, Contributed review: Optical micro- and nanofiber pulling rig, Rev. Sci. Instrum. 85, 111501 (2014).

[32] P. Anderson, S. Jalnapurkar, E. S. Moiseev, D. Chang, P. E. Barclay, A. Lezama, and A. I. Lvovsky, Optical nanofiber temperature monitoring via double heterodyne detection, AIP Adv. 8, 055005 (2018).

[33] R. P. Abel, A. K. Mohapatra, M. G. Bason, J. D. Pritchard, K. J. Weatherill, U. Raitzsch, and C. S. Adams, Laser frequency stabilization to excited state transitions using electromagnetically induced transparency in a cascade system, Appl. Phys. Lett. 94, 071107 (2009).

[34] D. Grischkowsky, Coherent excitation, incoherent excitation, and adiabatic states, Phys. Rev. A 14, 802 (1976).

[35] V. G. Minogin and S. Nic Chormaic, Manifestation of the van der Waals surface interaction in the spontaneous emission of atoms into an optical nanofiber, Laser Phys. 20, 32 (2010).

[36] M. Frawley, S. Nic Chormaic, and V. Minogin, The van der Waals interaction of an atom with the convex surface of a nanocylinder, Phys. Scr. 85, 058103 (2012).

[37] B. D. Patterson, P. Solano, P. S. Julienne, L. A. Orozco, and S. L. Rolston, Spectral asymmetry of atoms in the van der Waals potential of an optical nanofiber, Phys. Rev. A 97, 032509 (2018).

[38] M. Marcuzzi, J. Minář, D. Barredo, S. de Léséleuc, H. Labuhn, T. Lahaye, A. Browaeys, E. Levi, and I. Lesanovsky, Facilitation Dynamics and Localization Phenomena in Rydberg Lattice Gases with Position Disorder, Phys. Rev. Lett. 118, 063606 (2017).

[39] K. Kamitani, T. Muranaka, H. Takashima, M. Fujiwara, U. Tanaka, S. Takeuchi, and S. Urabe, Measuring the charge density of a tapered optical fiber using trapped microparticles, Opt. Express 24, 4672 (2016).

[40] M. Harlander, M. Brownnutt, W. Hänsel, and R. Blatt, Trappedion probing of light-induced charging effects on dielectrics, New J. Phys. 12, 093035 (2010).

[41] D. P. Fahey and M. W. Noel, Excitation of Rydberg states in rubidium with near infrared diode lasers, Opt. Express 19, 17002 (2011).

[42] F. Camargo, R. Schmidt, J. D. Whalen, R. Ding, G. Woehl, S. Yoshida, J. Burgdörfer, F. B. Dunning, H. R. Sadeghpour, E. Demler, and T. C. Killian, Creation of Rydberg Polarons in a Bose Gas, Phys. Rev. Lett. 120, 083401 (2018). 